\title{
Investigation of adhesion forces at the peritoneal surface
}

\author{
G. W. Taylor ${ }^{1,2}$, T. W. Liskiewicz ${ }^{1}$, A. Morina ${ }^{1}$, A. Neville ${ }^{1}$, \\ P. Gaskell ${ }^{1}$ \& D. Jayne ${ }^{2}$ \\ ${ }^{1}$ School of Mechanical Engineering, University of Leeds, UK \\ ${ }^{2}$ Academic Department of Surgery, St. James' University Hospital, \\ Leeds, UK
}

\begin{abstract}
There have recently been great advances in technology for minimally invasive ('keyhole') abdominal surgery. The latest developments in this area are aimed at the miniaturisation of devices, with the ultimate goal of complete internalization within the abdominal cavity. In this respect, a method of reliable and safe adhesion to the peritoneum - the tissue that lines the abdominal wall and the abdominal organs - would be advantageous. This paper investigates the possibility of adhesion by capillary forces by opposing the wet peritoneal surface with a range of simple surfaces.
\end{abstract}

\section{Introduction}

Surgical practice has undergone major changes in the last two decades with the introduction of minimally invasive, or "keyhole" surgical techniques. The advantages of less pain, fewer scars, a quicker recovery, shorter hospital stay and improved cost-effectiveness have been seen for several operations (Perissat et al. [1]; Guillou [2]). While minimally invasive techniques for more complex procedures have been described and shown to be safe and effective, their true advantages are yet to be seen. The limitations are due to the inherent difficulty in performing complex procedures with current instrumentation which has very limited dexterity, ergonomics and visual feedback (Ballantyne [3]). These difficulties have led to the recent introduction of advanced technology in a number of different surgical specialties (Pott et al. [4]). However, while initial improvements have been seen, currently available systems for minimal access 
abdominal surgery are bulky, cumbersome and very expensive. As a result there has been a move towards miniaturisation of surgical technology (Kobayashi et al. [5]; Ikuta [6]), with the ultimate aim of completely internalised devices (Patronik et al. [7]; Rentschler et al. [8]).

Such trends in medical and surgical research have led to collaborations between mechanical engineers and surgeons, and the investigation of interfaces between synthetic engineered materials and human tissue (Menciassi [9];Kassim [10]). The particular tissue of interest to our group, with reference to the development of technology in abdominal surgery, is the peritoneum. This is a thin layer of cells that forms the internal lining of the abdomen. It covers the surfaces of every abdominal organ, and the internal surface of the abdominal wall and the diaphragm. The ability to adhere to this surface reliably, without causing damage, would be a great step forward in the advancement of technology for applications in minimally invasive surgery. The surface of the peritoneum is made up of a continuous single layer of flattened cells, upon which is a carpet of tiny projections known as microvilli. These projections are of similar dimensions and spacing (Gaudio et al. [11]) to previous biomimetic adhesive surfaces based on the adhesive surfaces geckos (Geim et al. [12]) and beetles (Cheung et al. [13]). Microvilli are typically around $2 \mu \mathrm{m}$ in length, and $100-150 \mathrm{~nm}$ in diameter, with variable spacing of between 1 and 3 per micron.

The aim of this paper is to investigate the possibility of adhesion by capillary forces by opposing the wet peritoneal surface with a range of synthetic surfaces. Such a capability would have a wide range of potential uses within minimally invasive surgical equipment.

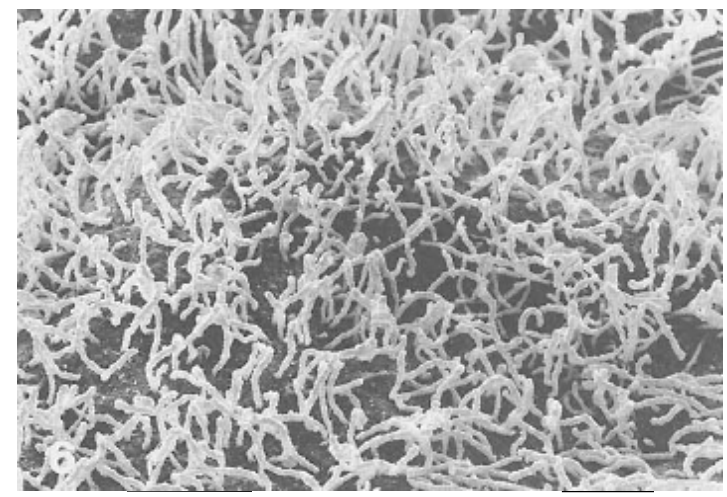

Figure 1: Scanning electron micrograph of rabbit peritoneum, showing

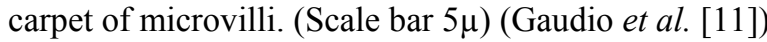

\section{Methods}

\subsection{Peritoneal samples}

Samples of abdominal wall were dissected immediately post mortem from mice and rats at University of Leeds Biomedical Services. The animals were bred in- 
house for research purposes other than this study. The samples were placed immediately in phosphate buffer solution (PBS). Experiments were carried out within 48 hours of tissue collection, and the tissue was refrigerated overnight. The peritoneal surface of the abdominal wall sample could be easily identified as it had much more prominent blood vessels near the surface. It is generally accepted that most mammalian abdominal tissues, including the peritoneum, are very similar to those of the human and can be used as proxy for this research.

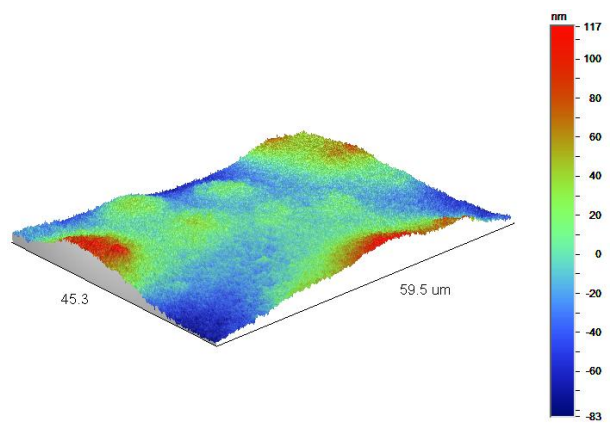

Figure 2: Three dimensional white light interferometer image of mouse peritoneum, showing a height range of $200 \mathrm{~nm}$. The three raised areas are likely to represent the areas overlying individual cell nuclei.

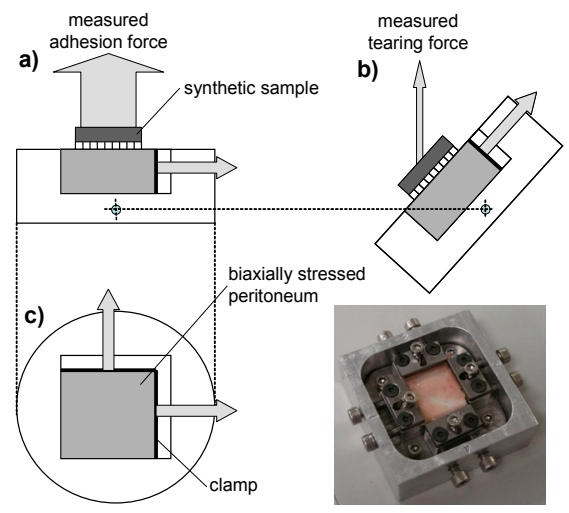

Figure 3: Experimental setup: a) adhesion force measurement; b) tearing force measurement; c) peritoneum holder.

\subsection{Experimental set-up}

The experimental set-up consisted of a platform and holding device for the peritoneal samples, a stub upon which to mount the synthetic materials and weights hung over a pulley to apply a vertical force. The peritoneum sample could be aligned at any angle to the horizontal to simulate the device being at any position on the insufflated abdomen. 
The adhesive quality of the peritoneum has been tested with a range of synthetic materials. A thin rubber square of $20 \times 20 \mathrm{~mm}$ was tested with mouse peritoneum, including the angle tests. Rat peritoneum was tested with a range of materials including rubber mounted on cork, polymer fabricated with moth-eye and lotus-leaf mimicking micro-patterns, "smooth surfaced" polymer, and a thin lacquer film. Most samples were $20 \times 20 \mathrm{~mm}$ squares, but the rubber with cork backing was tested across a range of surface areas. These latter tests were also repeated using a viscous detergent solution in order to investigate the effect of the viscosity of the surface fluid on adhesion.
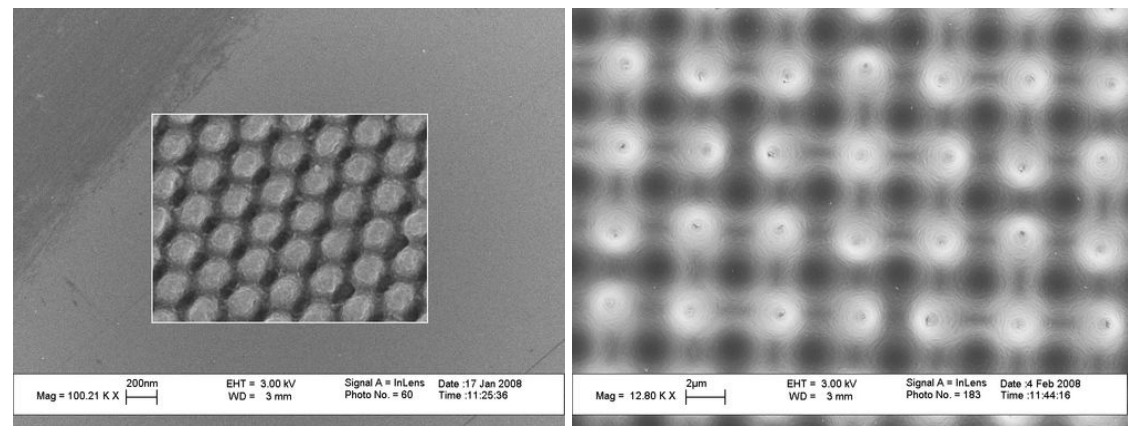

Figure 4: $\quad$ SEM images of synthetic surfaces: a) moth-eye like, b) lotus leaf like (Polymer samples courtesy of MacDermid).

\subsection{Procedure}

A tissue sample was cut into a $25 \mathrm{~mm}$ square, placed on the platform and held at its edges by clasps. These clasps were manually retractable, so biaxial stress could be placed on the sample to hold it tight, as well as stretch it to up to twice its size. Throughout the experiments the sample and its surface were prevented from drying by adding drops of PBS.

The synthetic materials were glued to aluminium stubs to be attached in turn to the pulley unit. The materials were brought down and placed by hand onto the peritoneal surface. To ensure immediate adhesion forces were being measured, the desired force was set up on the other side of the pulley prior to the surfaces coming into contact. Each material and condition was tested with a $5 \mathrm{~g}$ weight initially and then raised or lowered to find the maximum holding weight. Every time the weight was changed, the surfaces were separated, before being brought into contact again with the new weight. To qualify as a holding weight, the samples were required to remain in contact for at least 30 seconds.

For comparison the tests were repeated for the different materials, angles and surface area variables using rubber as the test surface in place of peritoneum. To maintain continuity, the surface was wetted with PBS, and prevented form drying throughout the experiments. 


\section{Results}

\subsection{Angle variables}

The loose rubber sample applied to the mouse peritoneal surface provided the most impressive results. A load of $200 \mathrm{~g}$ was suspended, and the test had to be abandoned at this point as the glue holding the rubber to the stub was failing. This was clearly a much greater adhesion than was seen when the same surface was mounted onto an inflexible cork backing and tested on rat peritoneum. It is unlikely that the mouse and rat peritoneum are so different as to have caused this effect, so it is likely that a different adhesion mechanism has been caused by different way of rubber sample attachment. The adhesion was much greater than in any of the other tests.

The loose rubber was also used to test the maximum angle at which adhesion could be maintained against a range of forces. Table 1 shows the inverse relationship between the adhesion force and the maximum angle at which that force can be maintained.

\subsection{Material variables}

Very similar adhesion forces were seen for each $20 \times 20 \mathrm{~mm}$ square applied to the rat peritoneum independent of the material used, with the exception of loose rubber. This was also the case for rubber as the test surface, but less adhesion was achieved for each surface (Table 2).

Table 1: Weight suspended to give vertical force versus maximum angle at which force is held by the contact (loose rubber to mouse peritoneum).

\begin{tabular}{|c|c|}
\hline Weight $(\mathrm{g})$ & Maximum angle (degrees) \\
\hline 25 & 48 \\
\hline 50 & 42 \\
\hline 75 & 24 \\
\hline 100 & 24 \\
\hline
\end{tabular}

Table 2: $\quad$ Material variables for rat peritoneum and rubber test surfaces.

\begin{tabular}{|c|c|c|}
\hline Material & \multicolumn{2}{|c|}{ Maximum weight suspended (g) } \\
\cline { 2 - 3 } (20x20mm squares) & Peritoneum & Rubber \\
\hline Loose rubber & 200 (abandoned) & 32 \\
\hline Rubber on cork backing & 7.5 & 6.5 \\
\hline Smooth surfaced polymer & 7.0 & 5.5 \\
\hline 'Motheye' patterned polymer & 6.5 & 5.0 \\
\hline 'Lotus' patterned polymer & 6.5 & 4.5 \\
\hline Thin, flexible lacquer film & 6.5 & 4.5 \\
\hline Rubber on foam backing & 7.5 & $<3$ \\
\hline
\end{tabular}




\subsection{Surface area variables}

There was a direct relationship between adhesion and surface area for both peritoneum and rubber test surfaces. However, the adhesion forces were less for rubber test surface (Fig. 5). Adhesion forces were also less when the peritoneal surface was treated with viscous detergent. The $13 \mathrm{~g}$ suspended for the $484 \mathrm{~mm}^{2}$ section of rubber would appear to be an anomaly. It may be that an edge was peeling away from its cork backing leading to a slight suction effect.

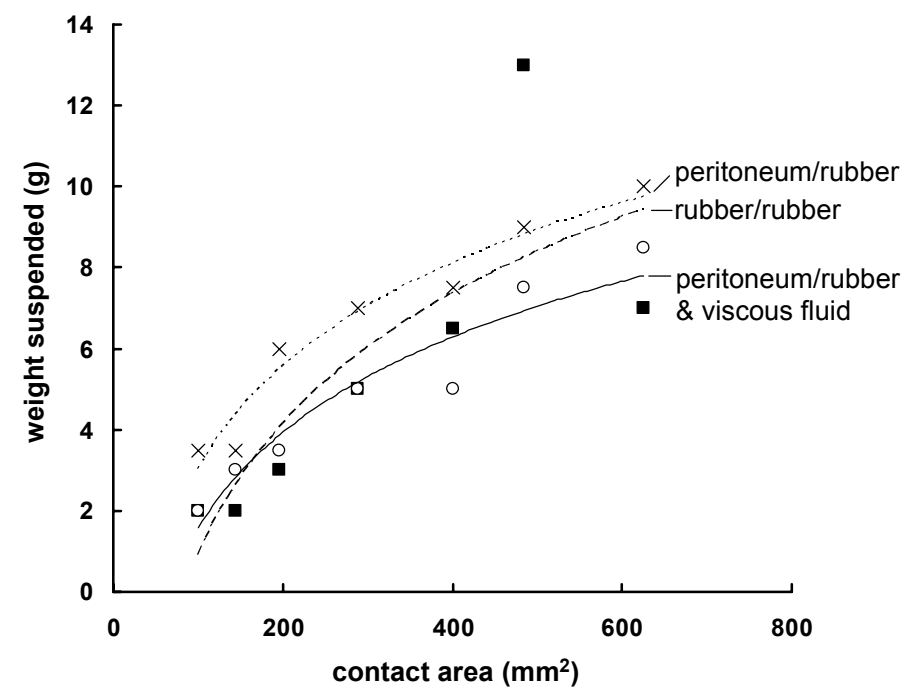

Figure 5: Evolution of suspended weight as a function of contact area for three different interface conditions.

\section{Discussion and conclusions}

The most notable finding from this study was that the loose section of rubber adhered far better than any other surface. The most likely explanation for this is that a suction force was set up as the vertical lifting force was concentrated on the centre of the section. All other samples apart from the thin plastic film were rigid or had a rigid backing and so the force was distributed across the surface. The plastic film differed from the rubber in that it had no elasticity and hence its central portion could not be lifted off the surface without a slight fold appearing and part of the edge becoming detached. Hence no suction force was possible with this arrangement.

However, other less dramatic but notable observations can also be made. The general trend throughout the tests was that greater adhesion was observed when peritoneum was used as the test surface as opposed to rubber. This included the surface area tests where rubber formed both sides of the tested interface. It would 
appear therefore that peritoneum has properties that enhance its adhesion. A possible explanation for this is that the microvilli on the surface play a part in increasing the surface area for contact, and allow an increased level of compliance with the opposing surface. This theory is supported by the observation that where the surface area of the opposing surface is reduced, such as on the moth-eye and lotus patterned polymers, the force of the adhesion is reduced.

It stands to reason that if capillary forces are acting an increase in total surface area in contact will increase the adhesion force. Further tests using materials that increase the available surface area are required to confirm this theory, and provide a basis for synthetic adhesion to the peritoneal surface.

\section{Acknowledgement}

The authors would like to thank the MacDermid Company for making the nanostructured polymer samples available for this study.

\section{References}

[1] Perissat, J., Collet, D., Belliard, R., Desplantez, J., \& Magne, E. 1992, "Laparoscopic cholecystectomy: the state of the art. A report on 700 consecutive cases", World J. Surg., vol. 16, no. 6, pp. 1074-1082.

[2] Guillou, P. J., Quirke, P., Thorpe, H., Walker, J., Jayne, D. G., Smith, A. M., Heath, R. M., \& Brown, J. M. 2005, "Short-term endpoints of conventional versus laparoscopic-assisted surgery in patients with colorectal cancer (MRC CLASICC trial): multicentre, randomised controlled trial", Lancet, vol. 365, no. 9472, pp. 1718-1726.

[3] Ballantyne, G. H. 2002, "Robotic surgery, telerobotic surgery, telepresence, and telementoring. Review of early clinical results", Surg. Endosc., vol. 16, no. 10, pp. 1389-1402.

[4] Pott, P. P., Scharf, H. P., \& Schwarz, M. L. 2005, "Today's state of the art in surgical robotics*”, Comput. Aided Surg., vol. 10, no. 2, pp. 101-132.

[5] Kobayashi Y., Chiyoda S., Watabe K., Masafumi O., \& Nakamura Y. 2007, "Small occupancy robotic mechanisms for endoscopic surgery." 5th International Conference on Medical Image Computing and Computer Assisted Intervention. LNCS, vol. 2488, pp. 75-82.

[6] Ikuta K., D. S. H. T. H. H. 2007, "Hyper-finger for remote minimally invasive surgery in deep area." Medical Image Computing and ComputerAssisted Intervention - MICCAI 2002: 5th International Conference, Tokyo, Japan.

[7] Patronik, N. A., Zenati, M. A., \& Riviere, C. N. 2005, "Preliminary evaluation of a mobile robotic device for navigation and intervention on the beating heart", Comput. Aided Surg., vol. 10, no. 4, pp. 225-232.

[8] Rentschler, M. E., Dumpert, J., Platt, S. R., Ahmed, S. I., Farritor, S. M., \& Oleynikov, D. 2006, "Mobile in vivo camera robots provide sole visual 
feedback for abdominal exploration and cholecystectomy", Surg. Endosc., vol. 20, no. 1, pp. 135-138.

[9] Menciassi, A., Stefanini, C., Orlandi, G., Quirini, M., \& Dario, P. 2006, "Towards active capsular endoscopy: preliminary results on a legged platform", Conf. Proc. IEEE Eng Med. Biol. Soc., vol. 1, pp. 2215-2218.

[10] Kassim, I., Phee, L., Ng, W. S., Gong, F., Dario, P., \& Mosse, C. A. 2006, "Locomotion techniques for robotic colonoscopy", IEEE Eng Med. Biol. Mag., vol. 25, no. 3, pp. 49-56.

[11] Gaudio, E., Casale, N., Pannarale, L., Priori, A., \& Marinozzi, G. 1990, “A scanning electron microscopy morphometric study of the rabbit peritoneal surface", Anat. Rec., vol. 228, no. 2, pp. 145-150.

[12] Geim, A. K., Dubonos, S. V., Grigorieva, I. V., Novoselov, K. S., Zhukov, A. A., \& Shapoval, S. Y. 2003, "Microfabricated adhesive mimicking gecko foot-hair", Nat. Mater., vol. 2, no. 7, pp. 461-463.

[13] Cheung, E., Karagozler, M. E., \& Sitti, M. 2005, "A New Endoscopic Microcapsule Robot using Beetle Inspired Microfibrillar Adhesives", Proceedings of the 2005 IEEE/ASME International Conference on Advanced Intelligent Mechatronics, vol. Monterey, California, USA, 24-28 July, 2005, pp. 551-557. 